\title{
Entwicklung eines unipolaren differentiellen Ladungsverstärkers für die Anwendung in eingebetteten Diagnosesystemen zur Druckmessung in Spritzgussmaschinen
}

\author{
M. Schneider ${ }^{1}$, A. Jahn 1 , N. Greifzu' ${ }^{1}$, N. Fränzel', \\ ${ }^{1}$ Hochschule Schmalkalden, Fakultät Elektrotechnik, \\ Blechhammer 6-9, 98574 Schmalkalden, Deutschland \\ ${ }^{2}$ Fraunhofer-Institut für Optronik, Systemtechnik und Bildauswertung, \\ Am Vogelherd 50, 98693 IImenau Deutschland
}

\section{Zusammenfassung}

Dieser Beitrag gibt einen Einblick in die Erarbeitung eines leistungsfähigen und kostengünstigen Ladungsverstärkers und zeigt eine mögliche Anwendung im Rahmen des Kunststoffspritzgießens. Mit einer Spannungsversorgung von 3,3 Volt und der Verwendung von Standardbauelementen wird eine Schaltung aufgezeigt, die ohne zusätzlichen Aufwand an handelsübliche Mikrocontroller angebunden werden kann. Durch die geringe Baugröße und die kleine Anzahl an verwendeten Bauelementen wird eine sehr kompakte und kosteneffiziente Lösung vorgestellt, die besonders auf das limitierte Platzangebot bei einer direkten Montage am Spritzgusswerkzeug gefordert sind.

Es wird gezeigt, dass der Verstärker in verschiedenen Laborversuchen, sowie auch im Fertigungsprozess gute Ergebnisse liefert. Des Weiteren wird ein Ansatz zur Datenfusion zwischen Kraft- und Drucksignalen mittels Kalman-Filter vorgestellt. Durch dieses Verfahren kann die Qualität der Sensorsignale deutlich verbessert werden.

Keywords: Piezoelektrische Drucksensoren, Ladungsverstärker, Chopper Amplifier (engl. Zerhack Verstärker), Kalman-Filter, Eingebettetes Diagnosesysteme

\section{Einleitung}

Zwei signifikante Faktoren der Preiskalkulation von Spritzgussartikeln beziehen sich auf die Fertigungsqualität und die Produktionsmengen. Gegenwärtig erfolgt die Aufzeichnung und Auswertung der Fertigungsparameter durch maschinenspezifische Hardware außerhalb des Spritzgusswerkzeugs. Diese Daten stehen dann der Maschine oder dem Rechnerleitsystem zur Verfügung. Dies setzt voraus, dass die Maschinen entsprechend ausgerüstet sind und das Unternehmen die nötige Infrastruktur besitzt. Im Falle eines Standortwechsels vom Werkzeughersteller zum Fertigungsbetrieb gehen bspw. oftmals die erarbeiteten Spritzgussparameter verloren.

Um den Informationsverlust und die daraus folgende Erhöhung der Umrüstzeit zu beseitigen, ist es erforderlich, ein eingebettetes System in das Werkzeug zu integrieren. Dieses System ermöglicht es, eine autonome Qualitätsüberwachung zu gewährleisten und somit auch die Sicherung der Fertigungseinstellungen zu übernehmen Es kann auch bei Firmen ohne Rechnerleitsystem verwendet werden.
Um ein solches Eingebettetes Diagnosesystem (EDS) für den Fertigungsbetrieb interessant zu machen, ist es notwendig, eine kostengünstige Hardware zu entwickeln. Der Fokus dieses Beitrags liegt auf der Entwicklung eines Ladungsverstärkers, der für den Einsatz in einem EDS zum Messen von piezoelektrischen Drucksignalen verwendet werden kann.

\section{Vorgehensweise}

Um ein solches Messsystem kosteneffektiv zu gestalten, wurden folgende Entwicklungsziele festgelegt:

1.) Für die Spannungsversorgung der Platinen und Sensoren soll eine geregelte Spannungsquelle mit $\mathrm{Vcc}=3,3 \mathrm{~V}$ verwendet werden.

2.) Der Bauraum der Auswerteeinheit mit Verstärkern muss möglichst gering gehalten werden.

3.) Die Kosten der verwendeten Verstärker für piezoelektrische Sensoren müssen verringert werden. 
Um den Anforderungen an Bauraum, Versorgungsspannung und Kostenminimierung gerecht werden zu können, wurden eigene Ladungsverstärker entwickelt und untersucht.

Erste Ergebnisse innerhalb der Forschungsund Entwicklungsarbeiten wurden mit einem Chopper-Verstärker realisiert. Dieser Verstärkeraufbau wurde in Anlehnung an [3] entwickelt. Der Verstärker arbeitet mit einer getakteten Referenzspannungsquelle am Eingangskondensator und ermöglicht es dadurch Ladungsänderungen in positive und negative Richtung messbar machen zu können [1]. Die folgende Abbildung 1 zeigt den schematischen Schaltplan des ChopperVerstärkers.

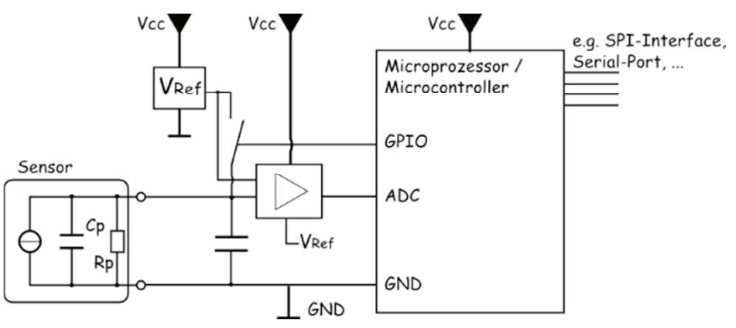

Abb. 1: Schema des Chopper-Verstärkers mit angeschlossenem Mikroprozessor und Ersatzschaltbild des piezoelektrischen Sensors

Mit Hilfe dieses Verstärkeraufbaus ist es möglich, auf kleinsten Raum eine ausreichend genaue Ladungsmessung durchführen zu können.

Der erarbeite Schaltplan wurde in ein Platinendesign überführt und gefertigt. Die folgende Abbildung 2 zeigt einen ca. $2 \mathrm{~cm} \times 3 \mathrm{~cm}$ kleinen Platinenausschnitt, welcher den Chopper-Verstärker darstellt.

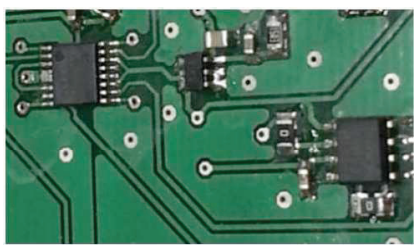

Abb. 2: Darstellung des Chopper-Verstärkers mit dem zugehörigen Platinenausschnitt (Abmessungen: ca. 2x3 $\mathrm{cm}$ )

Der Chopper-Verstärker arbeitet mit Hilfe einer getakteten Referenzspannungsquelle, welche das zu verstärkende Signal in kleine Abschnitte differenziert.

Dieses diskretisierte Signal gibt somit die Spannungs- / Ladungsänderung in einem bestimmten Zeitintervall wieder. Das verstärkte
Ableitungssignal wird an den Analog-DigitalWandler weitergeleitet und digitalisiert [2].

Um die Arbeitsweise dieses Verstärkers veranschaulichen zu können, wurde mit SCILAB $^{1}$ ein Simulationsprogramm entworfen. Die Grundlage dieser Simulation bildet ein periodisches Dreiecksignal, welches als Spannungssignal an den Chopper-Verstärker weitergegeben wird.

Die folgende Gleichung (kurz: GI.) (1) zeigt den analytischen Ausdruck dieses Testsignals im Zeitabschnitt $t_{0}=0 \mathrm{~s}$ bis $t=\mathrm{T} \quad$ ( $\mathrm{T}=$ Periodendauer) des Signals. Die GI. (2) ist das Ergebnis der Fourier-Reihenentwicklung des periodischen Dreiecksignals. Die Variable $\hat{u} / 2$ steht für den Maximalwert der eingestellten Spannung am Signalgenerator. Beide Gleichungen stellen den funktionalen Zusammenhang zwischen einem Spannungswert in Abhängigkeit über der Zeit dar. Die Variable $\omega_{0}$ steht für die Kreisfrequenz der jeweiligen Schwingung.

$$
\begin{aligned}
& u(t)=\left[\begin{array}{ll}
\hat{u} \cdot t & f \ddot{\mathrm{u}} r 0 \leq t \leq \frac{T}{2} \\
-\hat{u} \cdot t+\hat{u} T & f \ddot{\mathrm{u}} r \frac{T}{2} \leq t \leq T
\end{array}\right. \\
& u(t)=\frac{\widehat{u}}{2}-\frac{2 \hat{u}}{\pi^{2} f_{0}}\left(\cos \left(\omega_{0} t\right)+\frac{\cos \left(3 \omega_{0} t\right)}{3^{2}}+\cdots\right)
\end{aligned}
$$

Wenn man für $\hat{u}=\frac{2 \pi}{T}, T=1 s$ wählt, kann die Gl. (2) wie folgt umgeformt werden.

$$
u(t)=\pi-\frac{4}{\pi}\left(\cos (2 \pi t)+\frac{\cos (3 \cdot 2 \pi t)}{3^{2}}+\cdots\right)
$$

In Abbildung 3 sind zwei Perioden des Dreiecksignals dargestellt.

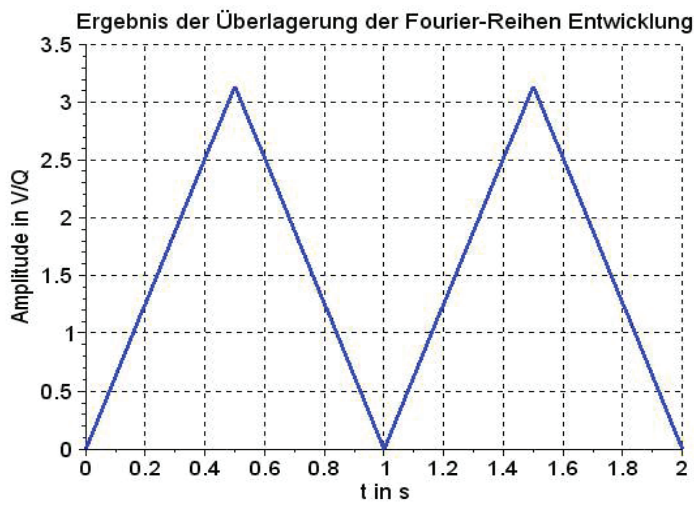

Abb.3: Darstellung des Dreiecktestsignals mit einer Periodendauer $T=1 \mathrm{~s}$ 
Die folgende Abbildung 4 veranschaulicht die Funktionsweise des Chopper-Verstärkers. Das Testsignal wird in periodischen Abständen (hier: 10ms) auf NULL gesetzt. Dadurch entsteht ein charakteristisches zerhacktes Signal, welches im Nachgang verstärkt wird.

Wie zu erkennen ist, wird durch die Funktionsweise des Chopper-Verstärkers eine Differentiation des Eingangssignals realisiert.

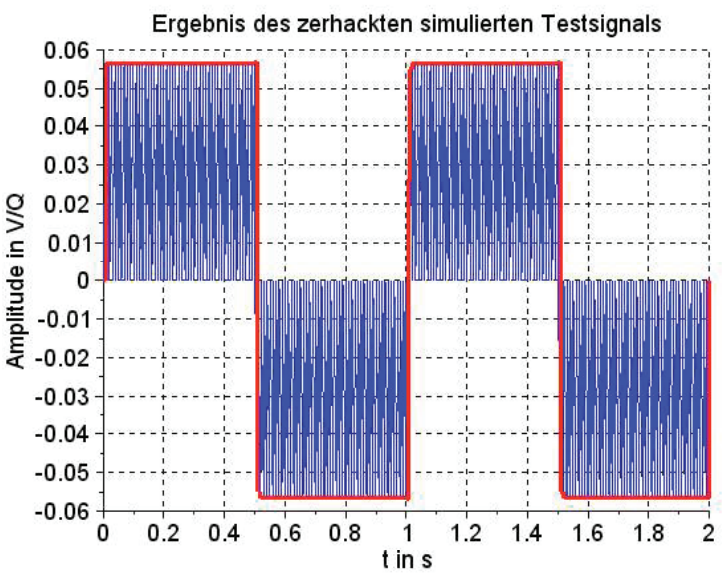

Abb.4: Darstellung des simulierten ChopperSignals (blau) mit der Überlagerung der ersten Ableitung des Dreiecksignals (rot).

Dieses Signal wird abgetastet und in digitale Werte umgewandelt. Um aus den digitalen Werten wieder das Eingangssignal herstellen zu können, müssen die digitalisierten Signale integriert werden. Durch die Integration des Chopper-Signals entsteht wieder das Testsignal (Dreiecktestsignal).

Um die Simulationsergebnisse überprüfen zu können, wurde ein Messaufbau mit Signalgenerator und Oszilloskop realisiert. Für die Umformung der vom Signalgenerator erzeugten Spannungssignale in eine Ladungsänderung, wurde eine speziell ausgemessene Kapazität in Reihenschaltung in den Signalweg eingesetzt. Eine Spannungsänderung an einer Kapazität (C) hat eine proportionale Änderung der Ladung zur Folge. Der mathematische Zusammenhang zwischen elektrischer Spannung (U) und elektrischer Ladung (Q) ist in GI. (4) dargestellt.

$$
Q=C \cdot U,(Q \sim U)
$$

Für die Testmessung mit dem Signalgenerator wurde eine Dreiecksignal mit einer Amplitude von $100 \mathrm{mV}$ mit einer Periodendauer von $\mathrm{T}=1 \mathrm{~s}$ an den entwickelten Ladungsverstärker angeschlossen.
Die angeschlossene Spannung führt an einer kalibrierten Kapazität mit $C=117,7 p F$, zu einer Ladungsänderung von $11,7 \mathrm{pC}$.

Das differenzierte und verstärkte Spannungssignal wurde mit einer Abtastrate von $1 \mathrm{kHz}$ digitalisiert. Der Nachweis der Simulationsergebnisse im Laborversuch wird im Abschnitt 3 beschrieben.

Für die Untersuchung des Temperaturverhaltens des Verstärkers wurde ein weiterer Messaufbau mit Hilfe einer Klimakammer durchgeführt. Die Abbildung 5 zeigt den schematischen Messaufbau.

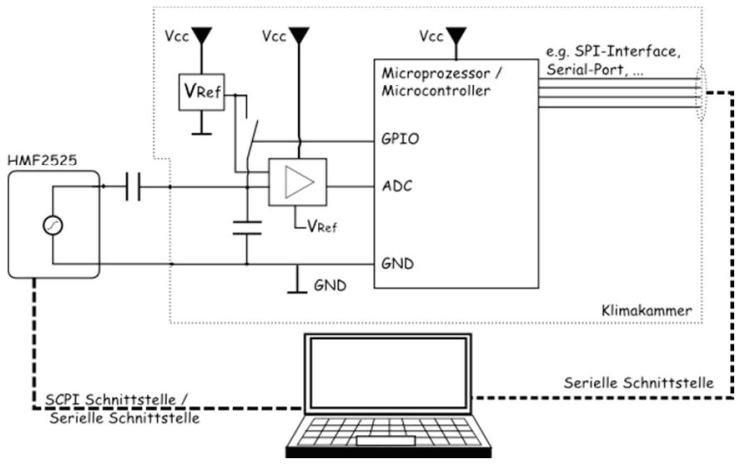

Abb.5: Schematische Darstellung eines vollautomatischen Messaufbaus mit Signalgenerator, Verstärkerschaltung und Datenauswertung mit Laptop

Für diese Messung wurden zwei Temperaturpunkte im Bereich der eingestellten Werkzeugtemperatur des Spritzgusswerkzeugs untersucht. Die eingestellten Umgebungstemperaturen in der Klimakammer betrugen $25^{\circ} \mathrm{C}$ und $50^{\circ} \mathrm{C}$.

Der Computer sendet die Einstellung für den HMF2525 Signalgenerator über eine SCPI Schnittstellen und empfängt gleichzeitig die gewandelten Werte des AD-Wandlers.

Die so aufgezeichneten Daten werden für die Bestimmung des Temperaturabhängigen Signalrauschabstandes und der Schwellwertbestimmung des Verstärkeraufbaus verwendet.

Um den Signalrauschabstand und die Empfindlichkeit des entwickelten Ladungsverstärkers bestimmen zu können, wurde der vollautomatische Messaufbau, wie in Abbildung 5 dargestellt, verwendet. Da die Angabe eines Signalrauschabstandes bei kommerziellen Ladungsverstärkern untypisch ist, ist dieser Abschnitt ist als Zusatzinformation für diesen speziellen Verstärker angegeben. Es soll dem Leser ein besseres Verständnis des Chopper-Verstärkers geben. Des Weiteren ermöglicht es mit der Schwellwertbestimmung eine qualitative und quantitative Bewertung der Güte des Ladungsverstärkers vorzunehmen.

Als Testsignale wurden mehrere Sinusschwingungen mit unterschiedlichen 
Frequenzen verwendet. Die Messreihe wurden mit einer Frequenz von $\mathrm{f}=1 \mathrm{~Hz}$ begonnen und in 19 Schritten bis $\mathrm{f}=2 \mathrm{OHz}$ durchgeführt.

Für die jeweilige Berechnung des Signalrauschabstandes werden die berechneten Werte der Spektralkoeffizienten des Eingangssignals, durch die Summe aller vom Eingangssignal unabhängigen Frequenzanteile dividiert. Der mathematische Zusammenhang hierfür ist in Gl. (5) abgebildet [6].

$$
S N R=20 \log \left(\frac{\text { Grundschwingung }}{\sqrt{\sum\left(\text { Rauschen }^{2}\right)}}\right)
$$

Ergebnisse zu diesen Messungen können im Abschnitt 4 nachgelesen werden.

Der Schwellwert der Schaltung ist die kleinste noch messbare Ladungsänderung (in $\mathrm{pC}$ ) die eine Änderung im least significant byte (LSB) des abgetasteten Spannungssignals erzeugt. Für diese Messung wird eine Rechteckspannung am Eingang des Verstärkers angelegt. Dieses Rechtecksignal führt durch das Verhalten des ChopperVerstärkers zu alternierenden periodischen nadelförmigen Impulsen (ähnlich DiracImpulsen). Die Ergebnisse dieser Messung werden im Abschnitt 5 erläutert.

Das Ziel der verschiedenen Untersuchungen ist ein Funktionstest im laufenden Spritzgießprozess. Hierfür wurde eine spezielle Messhardware angefertigt, die in das Spritzgießwerkzeug montiert wurde. Diese Hardware ermöglicht es, Daten der Drucksensoren und Kraftmessdosen parallel aufzuzeichnen und über eine serielle Schnittstelle an einen Empfangscomputer weiterzuleiten. Die detaillierte Beschreibung der Messhardware ist nicht Bestandteil des Beitrags.

Bei den zu fertigenden Spritzgussteilen handelt es sich um Zweikomponentenzugstäbe. Die Abbildung 6 zeigt das Spritzgusswerkzeug mit montierter Messhardware.

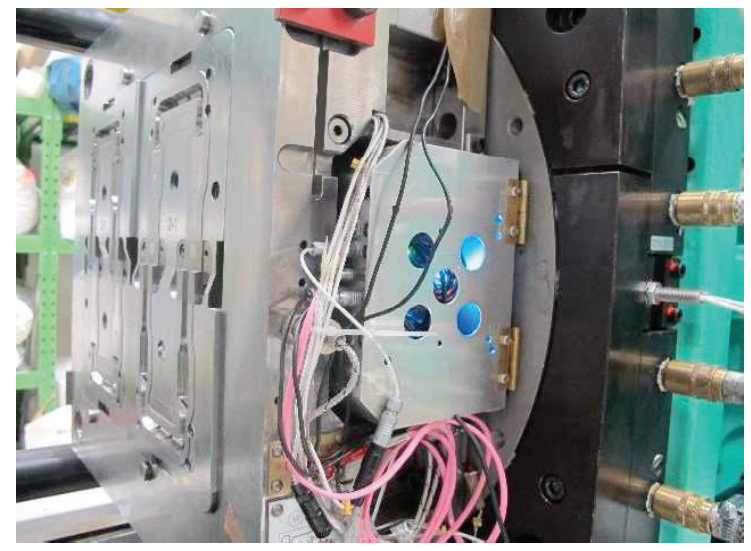

Abb.6: Fotographie des ZweikomponentenSpritzgusswerkzeugs mit montierter Messhardware
Die Messhardware ermöglicht es, die Sensoren mit einer Abtastrate von $1 \mathrm{kHz}$ maschinenunabhängig aufzuzeichnen, zu übertragen und auf dem Computer auszuwerten.

Die Implementierung der Kraftmessdosen wurde für die Überwachung der Auswerfer vorgesehen. In dem Messversuch dienen sie der Validierung der Druckergebnisse.

Für die Messungen im Spritzgusswerkzeug wurden Werkzeuginnendrucksensoren des Typ 6001A der Firma Priamus und Kraftsensoren KM26 der Firma ME-Meßsysteme GmbH verwendet.

Auswertung und Darstellung der Messergebnisse ist im Abschnitt 6 aufgeführt.

Um eine Verbesserung der Signalaufzeichnung realisieren zu können, wurden verschiedene Software Filter untersucht. Sehr gute Ergebnisse erzielte das Kalman-Filter.

Hierbei wird angenommen, dass am Eingang eines Blackbox-Modells die Drucksignale angelegt und durch das Verhalten des Systems in die Kraftsignale überführt werden.

Die Voraussetzung für den Kalman-Filter ist die Modellierung des Systems in der Zustandsraumdarstellung. Die Gleichungen (6) und (7) dienen hier als Grundgleichungen für das diskrete Zustandsraummodell [9].

$$
\begin{aligned}
& \underline{x}_{k}=\underline{A} \cdot \underline{x}_{k-1}+\underline{B} \cdot \underline{u}_{k}+\underline{w}_{k-1} ;(k \in N) \\
& \underline{y}_{k}=\underline{C} \cdot \underline{x}_{k}+\underline{v}_{k}
\end{aligned}
$$

Die Gleichung 6 wird auch als Prozess-Modell bezeichnet. Hieraus wird ersichtlich, dass die inneren Zustände $x_{k}$ des Systems nur von dem letzten Zustand aus der Vergangenheit $\underline{x}_{k-1}$ und von den Eingangsgrößen $\underline{u}_{k}$ abhängt.

Zusätzlich werden in den GI. (6) und (7) das Systemrauschen $\underline{w}_{k}$ und das Messrauschen $\underline{v}_{k}$ eingefügt. Das Systemrauschen $\underline{w}_{k}$ gibt an wie stark der Druckmesswert in das Ausgangssignal des Filters eingeht und wird als Standardabweichung des Systems ermittelt. Das Messrauschen $\underline{v}_{k}$ gibt an wie stark der Wert der Kraftmessung in das Ausgangssignal des Kalman-Filters eingeht. Mit Hilfe der Standardabweichung des Kraftsignals kann dieser Wert berechnet werden.

Das mit diesen Annahmen erstellte KalmanFilter, arbeitet hier als Zustandsbeobachter. Es versucht die inneren Zustände des Systems anhand einer Schätzung $\hat{x}_{k}$ anzunähern. Das Filter ist als eine parallele Struktur des realen Systems abgebildet. Die folgende Abbildung 7 veranschaulicht diesen Filter-Aufbau. 


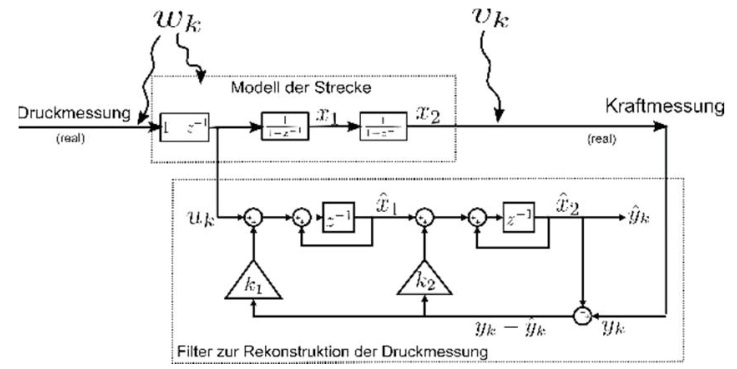

Abb.7: Darstellung des Systemmodells mit System- und Messrauschen und Abbildung der parallelen Filterstruktur [4]

Damit das Filter auf Abweichungen der Systemstrecke reagieren kann, werden der Ausgang $\underline{y}_{k}$ ständig mit dem Ausgang $\hat{y}_{k}$ des Filters verglichen. Die Differenz zwischen diesen Werten wird als Korrekturwert des geschätzten Zustandes $\hat{\underline{x}}_{k}$ des Filters verwendet.

Das erste Element im Streckenmodell bildet die Ableitung des Drucksignals. Wie in Abbildung 12 ersichtlich ist, sind die Rohdaten des Drucksensors um den Wert der Referenzspannung verschoben. Um diesen Offsetspannung eliminieren zu können, kann die Ableitung des Signals gebildet werden. Die beiden nachgelagerten Elemente dienen der Integration des Signals $\underline{u}_{k}$ und erzeugen mit dem System- und Messrauschen die Kraftkurve (siehe Abbildung 13).

Für die Berechnung der Filter Koeffizienten wird der rekursive Algorithmus des Kalman Filters verwendet. Die Erläuterung dieses mathematischen Zusammenhangs soll nicht Bestandteil dieses Beitrags sein. Die Abbildung 8 zeigt den erweiterten Aufbau des Filterentwurfs mit Ergänzung des rekursiven Algorithmus.

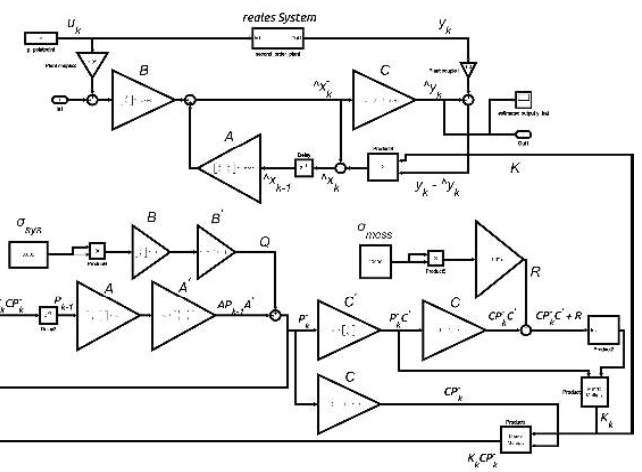

Abb.8: Erweiterte Darstellung des Filterentwurfs mit Ergänzung des rekursiven Kalman-Filters im unteren Teil [4]

\section{Verifikation der Simulationsergebnisse im Laborversuch}

Die Abbildung 9 zeigt das aufgezeichnete Dreieckstestsignal.

Wie man der Darstellung entnehmen kann, wird das erwartete Rechtecksignal mit einem Störsignal überlagert.

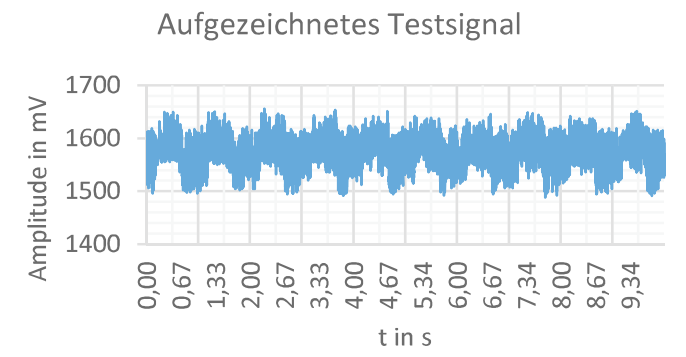

Abb.9: Darstellung des aufgezeichneten Dreiecksignals (Aufzeichnungsdauer 10s, Amplitude am Signalgenerator $10 \mathrm{mV}$, Periodendauer 1s) mit überlagerten $50 \mathrm{~Hz}$ Rauschen

Ursache für diese Störung kann zum größten Teil auf die $50 \mathrm{~Hz}$ Netzspannung zurückgeführt werden.

Wenn man von diesem Signal den Mittelwert abzieht und integriert, sollte wiederrum das Ursprungstestsignal entstehen. Die Abbildung 10 zeigt das Ergebnis der Integration.

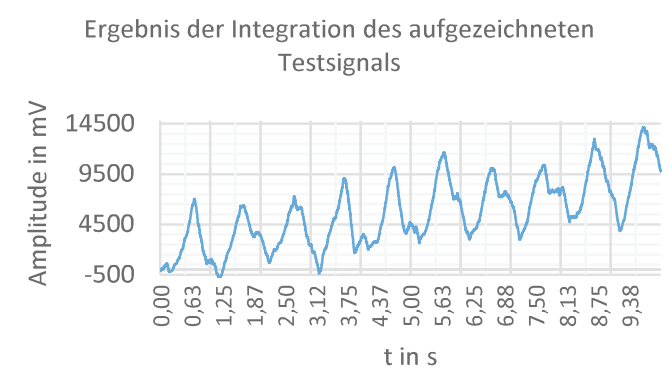

Abb.10: Ergebnis der Integration des verrauschten Testsignals. Darstellung der erwarteten Amplitude und Frequenz ist deutlich erkennbar

Wie man aus der Abbildung 10 erkennen kann, beträgt der verstärkte Spannungspegel ca. $5 \mathrm{~V}$ und die Periodendauer von $1 \mathrm{~s}$ ist deutlich abzulesen. 


\section{Ergebnisse der Untersuchung des Signalrauschabstands}

Der berechnete Signalrauschabstand (SNR) beträgt im Mittel bei T1 $=25^{\circ} \mathrm{C}$ um die $31,49 \mathrm{~dB}$ und bei $\mathrm{T} 2=50^{\circ} \mathrm{C}$ um die $30,96 \mathrm{~dB}$. Die Ergebnisse der Rauschmessungen für verschiedene Eingangsfrequenzen sind in Abbildung 11 dargestellt.

Wie man bei dieser Messung erkennen kann, gibt es drei Ausreißer, welche an unterschiedlichen Positionen im Graphen liegen.

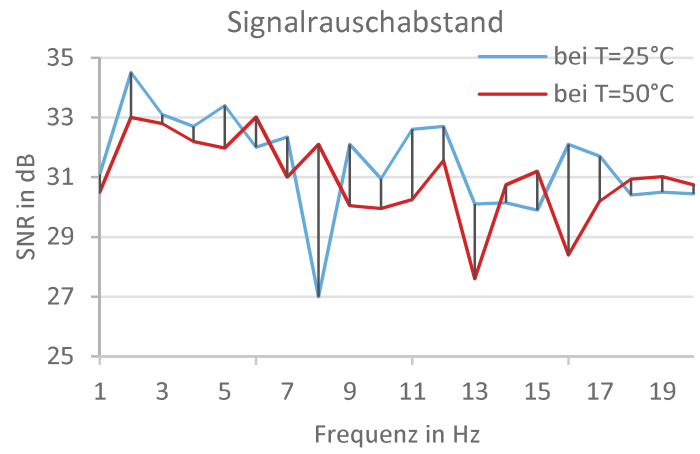

Abb.11: Messergebnis für den berechneten Signalrauschabstand in Abhängigkeit der Eingangsfrequenz und der Umgebungstemperatur [3]

\section{Ergebnisse der}

\section{Schwellwertbestimmung}

Die Ergebnisse dieser Messung können den Abbildung 12 und 13 entnommen werden. Wie zu erkennen ist, gibt es jeweils einen linearen Zusammenhang zwischen den Eingangssignalen und den gemessen Ausgangswerten. Wie man in beiden Abbildungen sieht, sind die Störpegel bei unterschiedlichen Umgebungstemperaturen nahezu konstant.

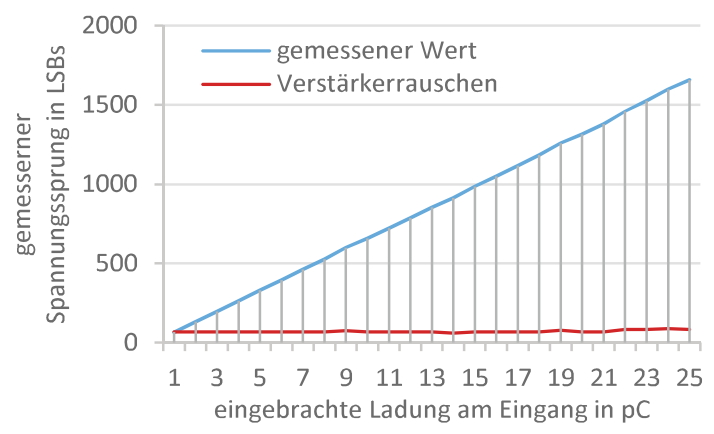

Abb.12: Schwellwertermittlung bei $25^{\circ} \mathrm{C}$ (Schnittpunkt der Graphen bei ca. 0.1pC) der Schaltung. Die blaue Kurve zeigt den Ausschlag in den Messdaten bei einer entsprechenden Eingangsladung. Der rote Graph zeigt das Dreifache des berechneten Verstärkerrauschens [3].

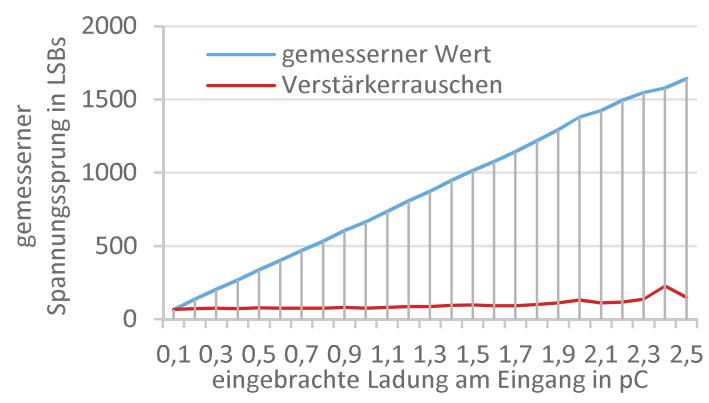

Abb.13: Schwellwertermittlung bei $50^{\circ} \mathrm{C}$ Umgebungstemperatur (Schnittpunkt der Graphen bei ca. 0.12pC) der Schaltung. Der blaue Verlauf zeigt den Ausschlag in den Messdaten bei einer entsprechenden Eingangsladung. Der Rote Graph zeigt das Dreifache des berechneten Verstärker-rauschens [3].

Als Grundlage zur Berechnung des Verstärkerrauschens wird der quadratische Mittelwert des Rauschens mit dem Faktor 3 multipliziert [7, S. 57]. Den Schwellwert des Ladungsverstärkers kann man am Schnittpunkt zwischen gemessenen Wert (blau) und Verstärkerrauschen (rot) ablesen. Er liegt etwa bei $0,1 \mathrm{pC} / \mathrm{LSB}$.

Nach [7, S. 209] besitzen kommerzielle Ladungsverstärker einen Schwellwert von 1fC.

\section{Ergebnisse der Messungen im Spritzgießprozess}

In den Abbildungen 14 und 15 sind die aufgezeichneten Sensorpegel während eines Spritzgießprozesses dargestellt. Die Aufzeichnungsdauer wurde auf 51 Sekunden begrenzt.

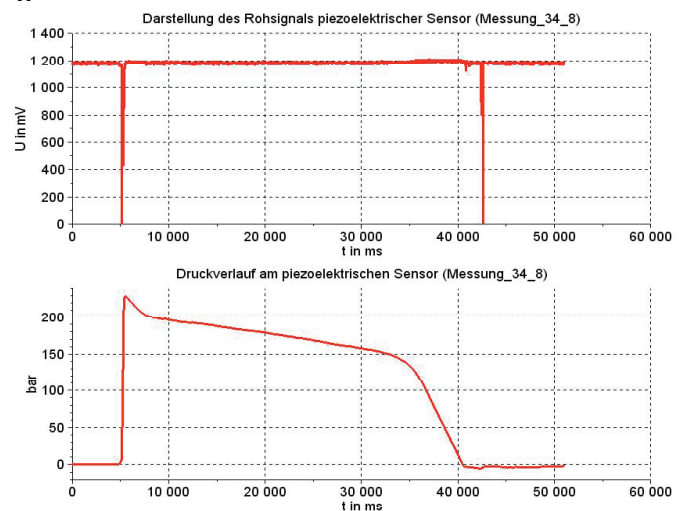

Abb.14: Oben: Darstellung des abgetasteten Chopper-Signals positive Druckänderungen ergeben einen negative Aussteuerung zur Referenzspannung. Unten: Betragsdarstellung des Integrierten Chopper-Signals nach Subtraktion der Referenzspannung (resultierender Druckverlauf am Priamus 6001A) 


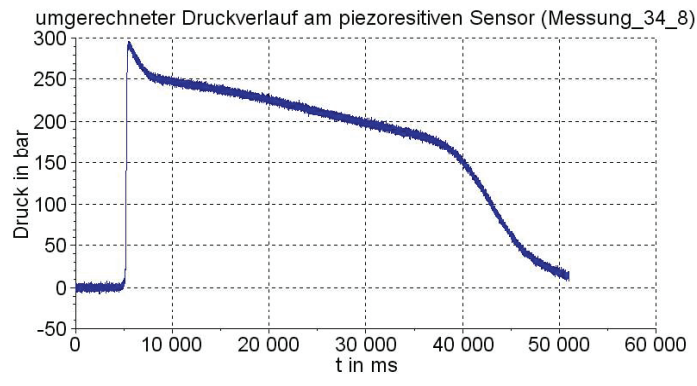

Abb.15: Darstellung des umgerechneten Druckverlaufs am KM26 Kraftsensor

Die Ursache für die negative Aussteuerung der Ladungskurve (oberer Abschnitt in Abbildung 14) ist dem geometrischen Aufbau des Piezosensors geschuldet. Durch die Vertauschung der Anschlüsse kann eine Spiegelung des Signals um die Referenzspannung realisiert werden.

Beide Messergebnisse spiegeln den gleichen Fertigungszyklus des Spritzgussprozesses wieder. Es ist festzustellen, dass beide Graphen einen ähnlichen Verlauf aufweisen. Ein gravierender Unterschied liegt in den aufgezeichneten Rauschpegeln der beiden Sensoren vor. Der Rauschpegel vom Kraftsensor ist um ein Vielfaches größer, als der des Integrierten Drucksignals. Des Weiteren ist ein deutlicher zeitlicher Verzug oberhalb einer Zeit von 40s zwischen den beiden des Sensors zu erkennen.

Die folgende Abbildung 16 zeigt 25 überlagerte Messungen des Chopper-Ladungsverstärkers.

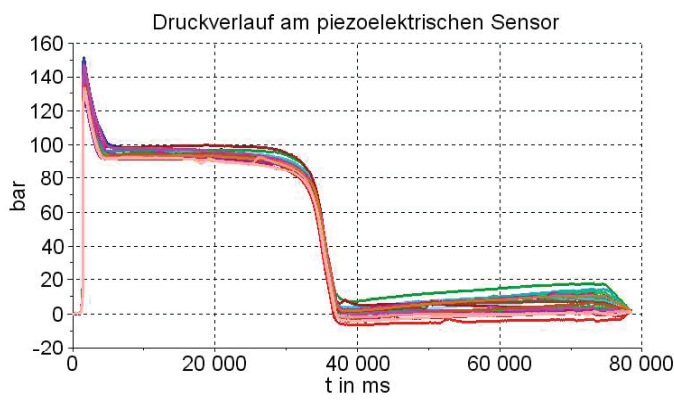

Abb.16: Darstellung von 25 Druckmessungen mit dem ChopperLadungsverstärkers, die Aufzeichnungsdauer betrug jeweils 78 Sekunden.

Wie man feststellen kann, kommt es beim Darstellen des Signalverlaufs des ChopperVerstärkers zu einen Drift in den Druckdaten. Diese Abweichungen sind oberhalb von 40s deutlich zu erkennen. Durch die parallele Aufzeichnung der Kraftsignale, können beide Messreihen direkt verglichen werden. In der Abbildung 17 sind keine Drifts der Sensorsignale ersichtlich, lediglich leichte
Amplitudenschwankungen zwischen den Messungen sind erkennbar.

Die Amplitudenänderungen jeweils am Ende der Messreihen im Kraftverlauf korrelieren mit den Driftänderungen im Drucksignal. Dies könnte auf das Auffahren des Spritzgusswerkzeugs hindeuten. Dieser Zusammenhang ist noch Gegenstand weiterer Untersuchungen.

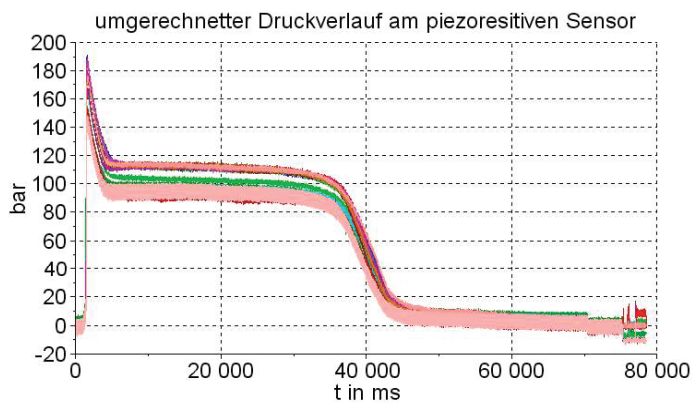

Abb.17: Darstellung von 25 Kraftmessungen in Druck umgerechnet, die Aufzeichnungsdauer betrug jeweils 78 Sekunden.

\section{Ergebnisse des Kalman-Filters}

Mit Hilfe dieses Filters ist es gelungen das Weglaufen des Drucksignals und die Störungen des Kraftsignals auszugleichen. Der rote Graph in Abbildung 18 zeigt das aufgezeichnete Kraftsignal ohne Filterung. Die schwarze Kurve ist das Ergebnis der fusionierten Sensorsignale.

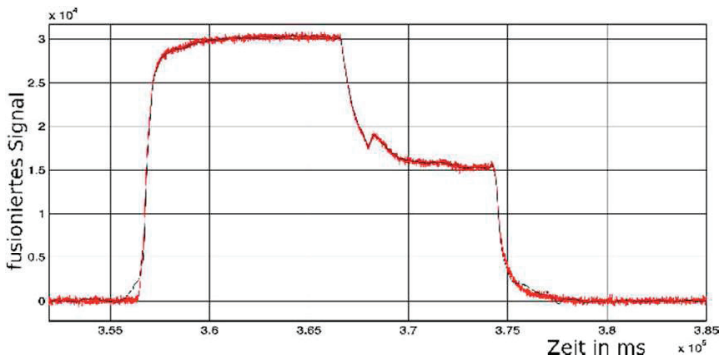

Abb.18: Darstellung des Kraftverlaufs eines Spritzgusszyklus (rot) mit dem Ergebnis der fusionierten / gefilterten Sensordaten (schwarz)

Wie man erkennen kann, besitzt das fusionierte Signal nahezu kein Rauschen und liegt meistens innerhalb des Rauschbandes der Kraftkurve.

\section{Schlussfolgerungen}

Wie man aus den Ergebnissen im Abschnitt 3 entnehmen kann, konnten die Störeinflüsse der Netzspannung durch die Integration des Signals eliminiert werden. Jedoch ist zu erkennen, dass ein leicht ansteigender Spannungsverlauf im Ergebnissignal entsteht. Dieser Spannungsdrift ist voraussichtlich auf einem Integrationsfehler durch die 
Störspannungen und die kurzen "Reset"Phasen beim Zerhacken des Eingangssignals zurückzuführen.

Die Störungen während der Bestimmung des Signalrauschabstands fallen zeitlich mit dem Anlaufen eines fest installierten Lüfters in der Klimakammer zusammen. Die Motoren der Lüfter erzeugen in einem anderen Spektralabschnitt einen Stör-Peak, welcher das SNR um bis zu $4 \mathrm{~dB}$ verschlechtern kann. Dieser Effekt wird im weiteren Verlauf der Forschungsarbeiten durch eine bessere elektrische Schirmung der Elektronik verringert. Der im Abschnitt 5 ermittelte Schwellwert liegt etwa um den Faktor 100 schlechter als bei kommerziellen Ladungsverstärkern, jedoch besitzen diese erheblichen größere Abmessungen, einen deutlich höheren Versorgungsspannung und die meisten Piezosensoren besitzen eine Genauigkeit von $10 \mathrm{pC} / \mathrm{bar}$. Aus diesen Grund stellt sich die Frage: Ist es überhaupt sinnvoll, hochgenaue Verstärkertechnik im Spritzgießprozess zu verwenden?

Mit den Messungen im laufenden Fertigungsprozess konnte der Verstärker auf seine Funktionsfähigkeit getestet werden. Die im Abschnitt 6 beschrieben Unterschiede im zeitlichen Verlauf zwischen Druck- und Kraftsignale können an der unterschiedlichen Position der Sensoren in der Kavität [8] oder dem an Kraftsensor montierte Auswerfmechanismus liegen. Jedoch liefert der entwickelte Ladungsverstärker im Fertigungsprozess robuste und saubere Ergebnisse mit einer ausreichend hohen Auflösung.

Mit Hilfe des erarbeiten Kalman-Filters konnte gezeigt werden, dass eine Datenfusion zwischen Kraft und Drucksignalen möglich ist und eine Verbesserung der Signalqualität realisiert werden konnte. Die zuvor gemessenen Drifterscheinungen im Drucksignal konnten durch die Datenfusion eliminiert werden. Des Weiteren konnte das Rauschen des Kraftsignals nahezu beseitigt werden. Weitere Untersuchungen der Datenfusion sind noch Gegenstand der Forschung.

Mit diesen Beitrag konnte gezeigt werden, dass der entwickelte Verstärker für die Ladungsmessung von piezoelektrischen Sensoren gute Eigenschaften besitzt und in Eingebetteten System eingesetzt werden kann.

\section{Danksagung}

Besonderer Dank gilt der Thüringer Aufbaubank als Projektträger für die Förderung dieses Forschungsprojekts. Die Ergebnisse dieser zugrundeliegenden Arbeit wurde mit Mitteln des Freistaates Thüringen und der Europäischen Union (ESF) unter dem Förderkennzeichen 2013 FRG 0130 gefördert. Die Verantwortung für den Inhalt dieser Veröffentlichung liegt bei den Autoren.

\section{Literaturnachweis}

[1] A. Jahn, Konzeption, Umsetzung und Test einer analogen Signalaufbereitungsschaltung für piezoelektrische Drucksensoren, Bachelorarbeit Hochschule Schmalkalden 2015

[2] N. Greifzu, Entwicklung von Hard- und Software zur Messung von Kraft-, Druck- und Temperatursignalen in Kunststoffspritzgussmaschinen, Masterarbeit Hochschule Schmalkalden 2015

[3] C. C. Enz, G. C. Temes, Circuit techniques for reducing the effects of op-amp imperfections: autozeroing, correlated double sampling, and chopper stabilization, Proceedings of the IEEE 84.11 (Nov 1996), dio: 10.1109/5.542410

[4] P. Symons, Digital Waveform Generation, Cambridge University Press 2013, S. 321, 9781107496453

[5] G. Gautschi, Piezoelectric Sensorics - Force Strain, Pressure, Acceleration and Acoustic Emission Sensors Materials, Springer 2002, 978-3-662-04732-3

[6] W. T. JR. Higgins, A Comparison of Complementary and Kalman Filtering, IEEE TRANSACTION ON AEROSPACE AND ELECTRONIC SYSTEMS 11.3 (Mai 1975), S 321 - 325, dio: 10.1109/TAES.1975.308081

[7] G. Welch, G. Bishop, An Introduction to the Kalman Filter, University of North Carolina, Department of Computer Science, TR 95-041

[8] M. Schneider, A. Wenzel, Entwurf eines Eingebetteten Diagnosesystems zur Überwachung von Prozessparametern beim Spritzgießen, Tag der Forschung FHS-prints 2/2014 S. 91 - 104, ISSN: 0949-1767 